\title{
Komar integrals for theories of higher order in the Riemann curvature and black-hole chemistry
}

\author{
Tomás Ortín \\ Instituto de Física Teórica UAM/CSIC, \\ C/ Nicolás Cabrera, 13-15, C.U. Cantoblanco, E-28049 Madrid, Spain \\ E-mail: tomas.ortin@csic.es
}

ABSTRACT: We construct Komar-type integrals for theories of gravity of higher order in the Riemann curvature coupled to simple kinds of matter (scalar and vector fields) and we use them to compute Smarr formulae for black-hole solutions in those theories. The equivalence between $f(R)$ and Brans-Dicke theories is used to argue that the dimensionful parameters that appear in scalar potentials must be interpreted as thermodynamical variables (pressures) and we give a general expression for their conjugate potentials (volumes).

KEYwords: Black Holes, Classical Theories of Gravity

ArXiv EPrint: 2104.10717 


\section{Contents}

1 Derivation of the Komar charge 2

2 Examples $\quad 6$

2.1 General relativity in presence of a cosmological constant 6

2.2 Lovelock gravities 6

2.3 Dilaton gravity 8

2.3.1 Cosmological Reissner-Nordström-Tangherlini black holes 9

$\begin{array}{lll}2.4 & f(R) \text { gravity } & 11\end{array}$

3 Discussion 14

$\begin{array}{ll}\text { A Determination of } J^{\alpha \mu} & 16\end{array}$

\section{Introduction}

Komar integrals [1] provide a simple and economic way of computing the mass of spacetimes admitting a timelike Killing vector, but, as shown in ref. [2], they can also be used to obtain Smarr formulae [3] relating the mass to the conserved charges. Since the Smarr formula can be obtained from the first law of black-hole mechanics, the terms that occur in it have a direct thermodynamical interpretation. ${ }^{1}$ This relation and the presence of the cosmological constant in the Smarr formula $[8,9]$ hinted at the interpretation of the cosmological constant as a thermodynamical "pressure" with a conjugate thermodynamical potential ("volume") which, in its turn, means that the mass should be interpreted as an enthalpy rather than as an internal energy. This realization, extended to other dimensionful parameters occurring in the action such as the coefficients of the Lovelock terms [2], has lead to the discovery of a host of new phenomena involving black holes, opening a new field that has been named black-hole chemistry. ${ }^{2}$

The coupling to matter has not been considered in most of these developments. In particular, no Komar integrals have been proposed for theories with matter fields, even though one simply has to follow the recipe of ref. [4] to construct them systematically with the Noether charge. ${ }^{3}$ One of the reasons may be that the treatment of matter fields in refs. [5-7] is not valid for matter fields that have some kind of gauge freedom (all matter fields but uncharged scalars, as a matter of fact), which leads to Noether charges which are not gauge-invariant, for instance.

\footnotetext{
${ }^{1}$ As shown in ref. [4], Komar formulae can be obtained from the diffeomorphisms Noether charge using the methods of refs. [5-7].

${ }^{2}$ For reviews with many references see refs. [10, 11].

${ }^{3}$ The recipe of ref. [4] leads to a combination of surface and volume integrals, but, as we will see, the volume integrals can always be rewritten as surface integrals.
} 
In this paper, in section 1, we use the treatment of fields with gauge freedoms proposed in refs. [12-14] to find the Noether charge and to construct explicit expressions for the Komar integral in theories of gravity of higher order in the Riemann curvature minimally coupled to a Maxwell field (see also [15].) Additional fields of different kinds can be treated in exactly the same way and we will work out in section 2 a few examples: General Relativity in presence of a cosmological constant in section 2.1, Lovelock gravities (studied in [2]) in section 2.2, dilaton gravity in section 2.3 and $f(R)$ gravities in section 2.4. In particular, and as a test of our formulae, we will obtain the Smarr formula for cosmological Reissner-Nordström-Tangherlini black holes (more often known as $d$-dimensional ReissnerNordström-(anti-)De Sitter black holes) in section 2.3.1.

The example of $f(R)$ gravities is interesting and tractable. The action of these theories contains a series of higher-order terms weighted by a dimensionful parameter, as in general Lovelock gravities, which end up in the Smarr formula. By analogy with the Lovelock case, it is natural to interpret those parameters as thermodynamical variables ("pressures", again). The equivalence between $f(R)$ and Brans-Dicke theories with a scalar potential determined by the dimensionful parameters suggests that, in a general theory with a scalar potential, the dimensionful parameters that define it must have the same interpretation. It is, then, tempting to extend the analogy to all the parameters that define the theory as a deformation of the simplest one [16]. We will discuss this proposal and future work in section 3.

\section{Derivation of the Komar charge}

We are interested in general, diffeomorphism and gauge-invariant $d$-dimensional, theories of metric gravity minimally coupled to arbitrary matter fields which, in particular, can have gauge freedoms. However, for the sake of simplicity, we will just consider a Maxwell field $A_{\mu}$ with field strength $F_{\mu \nu}=2 \partial_{[\mu} A_{\nu]}$, which suffices to illustrate the treatment of the gauge transformations. We also set the overall factor of $16 \pi G_{N}^{(d)}=1$ for the moment. Thus, we consider the action

$$
S[g, A]=\int d^{d} x\left\{\mathcal{L}_{\text {grav }}-\frac{1}{4} \sqrt{|g|} F^{2}\right\} .
$$

We assume that $S_{\text {grav }}[g]$ contains terms of arbitrary order in the Riemann curvature tensor contracted using the metric. ${ }^{4}$

Under an arbitrary variation of the fields, the action behaves as

$$
\delta S=\int\left\{\frac{\delta S}{\delta g_{\mu \nu}} \delta g_{\mu \nu}+\frac{\delta S}{\delta A_{\mu}} \delta A_{\mu}+\partial_{\mu} \Theta^{\mu}\left(g, \delta_{\xi} g, A, \delta_{\xi} A\right)\right\} .
$$

\footnotetext{
${ }^{4}$ In our conventions, the Riemann tensor is defined as

$$
R_{\mu \nu \rho}^{\sigma} \equiv 2 \partial_{[\mu} \Gamma_{\nu] \rho}^{\sigma}+2 \Gamma_{[\mu \mid \lambda}^{\sigma} \Gamma_{\mid \nu] \rho}^{\lambda},
$$

where $\Gamma_{\mu \nu}{ }^{\rho}$ is the Levi-Civita connection, whose components are the Christoffel symbols

$$
\Gamma_{\mu \nu}{ }^{\rho}=\left\{\begin{array}{c}
\rho \\
\mu \nu
\end{array}\right\} \equiv \frac{1}{2} g^{\rho \sigma}\left\{\partial_{\mu} g_{\nu \sigma}+\partial_{\nu} g_{\mu \sigma}-\partial_{\sigma} g_{\mu \nu}\right\} .
$$


Using the formalism of ref. [17], ${ }^{5}$ the equations of motion and the total derivative can be written in the form

$$
\begin{aligned}
\frac{\delta S}{\delta g_{\mu \nu}} & =\frac{1}{2} g^{\mu \nu} \mathcal{L}_{\text {grav }}-P^{\mu \alpha \beta \gamma} R_{\alpha \beta \gamma}^{\nu}+2 \nabla_{(\alpha} \nabla_{\beta)} P^{\alpha \mu \beta \nu}+\frac{1}{2} \sqrt{|g|} T^{\mu \nu}, \\
\frac{\delta S}{\delta A_{\mu}} & =\partial_{\mu}\left(\sqrt{|g|} F^{\mu \nu}\right), \\
\Theta^{\mu}(g, \delta g, A, \delta A) & =2 P^{\mu \alpha \beta} \delta \Gamma_{\alpha \beta}^{\gamma}-2 \nabla_{\gamma} P^{\gamma \alpha \mu \beta} \delta g_{\alpha \beta}-\sqrt{|g|} F^{\mu \nu} \delta A_{\nu} .
\end{aligned}
$$

with

$$
\begin{aligned}
P^{\mu \nu \rho \sigma} & \equiv \frac{\partial \mathcal{L}_{\text {grav }}}{\partial R_{\mu \nu \rho \sigma}}, \\
T^{\mu \nu} & \equiv F^{\mu \alpha} F^{\nu}{ }_{\alpha}-\frac{1}{4} g^{\mu \nu} F^{2}, \\
\delta \Gamma_{\alpha \beta}{ }^{\gamma} & =\frac{1}{2} g^{\gamma \delta}\left\{\nabla_{\alpha} \delta g_{\beta \delta}+\nabla_{\beta} \delta g_{\alpha \delta}-\nabla_{\delta} \delta g_{\alpha \beta}\right\} .
\end{aligned}
$$

It follows from its definition that $P^{\mu \nu \rho \sigma}$ shares the symmetries of the Riemann tensor which, in particular, means that

$$
P^{[\mu \nu \rho] \sigma}=0
$$

Another important property is

$$
P^{[\mu \mid \alpha \beta \gamma} R_{\alpha \beta \gamma}^{\mid \nu]}=0 .
$$

Now, we specialize this general expression to the case of diffeomorphisms generated by an infinitesimal vector field $\xi^{\mu}$. The transformations of the fields are given by

$$
\begin{aligned}
\delta_{\xi} g_{\mu \nu} & =-£_{\xi} g_{\mu \nu}=-2 \nabla_{(\mu} \xi_{\nu)}, \\
\delta_{\xi} A_{\mu} & =-\mathbb{L}_{\xi} A_{\mu},
\end{aligned}
$$

where $\mathbb{L}_{\xi}$ is a generalization of the standard Lie derivative $£_{\xi}$ that takes into account the gauge freedoms of the matter fields and which is a combination of the standard Lie derivative $£_{\xi}$ and "compensating" gauge transformations with $\xi$-dependent gauge parameters $\chi_{\xi}$, $\delta_{\chi_{\xi}} \cdot{ }^{6}$ In the case of a Maxwell field,

$$
\chi_{\xi}=\xi^{\mu} A_{\mu}-P_{\xi},
$$

where $P_{\xi}$ is such that, when $\xi$ generates a symmetry of the whole field configuration (in particular, it is a Killing vector), it satisfies the momentum-map equation

$$
\partial_{\mu} P_{k}=-k^{\nu} F_{\mu \nu} .
$$

\footnotetext{
${ }^{5}$ See also ref. [15] for many results related to higher-order theories, in particular in relation to Wald's formalism.

${ }^{6}$ See ref. [12] and references therein.
} 
Then,

$$
\begin{aligned}
\mathbb{L}_{\xi} A_{\mu} & =£_{\xi} A_{\mu}-\delta_{\chi_{\xi}} A_{\mu}=\xi^{\nu} \partial_{\nu} A_{\mu}+\partial_{\mu} \xi^{\nu} A_{\nu}-\partial_{\mu}\left(\xi^{\nu} A_{\nu}-P_{\xi}\right) \\
& =\xi^{\nu} F_{\nu \mu}+\partial_{\mu} P_{\xi},
\end{aligned}
$$

which vanishes identically for $\xi^{\mu}=k^{\mu}$ by virtue of the momentum-map eq. (1.11).

Substituting these variations in eq. (1.4) we get

$$
\delta_{\xi} S=-\int\left\{2 \frac{\delta S}{\delta g_{\mu \nu}} \nabla_{\mu} \xi_{\nu}+\frac{\delta S}{\delta A_{\mu}}\left(\xi^{\nu} F_{\nu \mu}+\partial_{\mu} P_{\xi}\right)-\partial_{\mu} \Theta^{\mu}\left(g, \delta_{\xi} g, A, \delta_{\xi} A\right)\right\}
$$

where

$$
\begin{aligned}
\Theta^{\mu}\left(g, \delta_{\xi} g, A, \delta_{\xi} A\right) \equiv & 2 P^{\mu \alpha \beta} \delta \Gamma_{\alpha \beta} \gamma-2 \nabla_{\gamma} P^{\gamma \alpha \mu \beta} \delta g_{\alpha \beta}-\sqrt{|g|} F^{\mu \nu} \delta A_{\nu} \\
= & -4 P^{\mu \alpha \beta \gamma} \nabla_{\beta} \nabla_{(\alpha} \xi_{\gamma)}+4 \nabla_{\gamma} P^{\gamma \alpha \mu \beta} \nabla_{(\alpha} \xi_{\beta)} \\
& +\sqrt{|g|} F^{\mu \alpha} F_{\nu \alpha} \xi^{\nu}+\sqrt{|g|} F^{\mu \nu} \partial_{\nu} P_{\xi}
\end{aligned}
$$

Integrating by parts the first term and using the identity

$$
\nabla_{\mu} \frac{\delta S}{\delta g_{\mu \nu}}=\frac{1}{2} \sqrt{|g|} \nabla_{\mu} T^{\mu \nu}=\frac{1}{2} \frac{\delta S}{\delta A_{\alpha}} F_{\nu \alpha},
$$

we get

$$
\delta_{\xi} S=-\int\left\{\frac{\delta S}{\delta A_{\mu}} \partial_{\mu} P_{\xi}-\partial_{\mu}\left[\Theta^{\mu}\left(g, \delta_{\xi} g, A, \delta_{\xi} A\right)-2 \frac{\delta S}{\delta g_{\mu \nu}} \xi^{\nu}\right]\right\} .
$$

Integrating by parts again and using now the Noether identity associated to the invariance under the gauge transformations $\delta_{\chi} A_{\mu}=\partial_{\mu} \chi$, namely

$$
\partial_{\mu} \frac{\delta S}{\delta A_{\mu}}=0
$$

we arrive at

$$
\delta_{\xi} S=\int \partial_{\mu}\left[\Theta^{\mu}\left(g, \delta_{\xi} g, A, \delta_{\xi} A\right)-2 \frac{\delta S}{\delta g_{\mu \nu}} \xi_{\nu}-\frac{\delta S}{\delta A_{\mu}} P_{\xi}\right] .
$$

Finally, the invariance of the action under diffeomorphisms means that

$$
\delta_{\xi} S=-\int d^{d} x \partial_{\mu}\left(\xi^{\mu} \mathcal{L}\right)
$$

and we arrive at the following off-shell identity:

$$
\partial_{\mu} J^{\mu}=0
$$

where

$$
\begin{aligned}
J^{\mu}= & \Theta^{\mu}\left(g, \delta_{\xi} g, A, \delta_{\xi} A\right)-2 \frac{\delta S}{\delta g_{\mu \nu}} \xi_{\nu}-\frac{\delta S}{\delta A_{\mu}} P_{\xi}+\xi^{\mu} \mathcal{L} \\
= & -4 P^{\mu \alpha \beta \gamma} \nabla_{\beta} \nabla_{(\alpha} \xi_{\gamma)}+4 \nabla_{\gamma} P^{\gamma \alpha \mu \beta} \nabla_{(\alpha} \xi_{\beta)}+2 P^{\mu \alpha \beta \gamma} R_{\alpha \beta \gamma}^{\nu} \xi_{\nu}-4 \nabla_{(\alpha} \nabla_{\beta)} P^{\alpha \mu \beta \nu} \xi_{\nu} \\
& -\partial_{\nu}\left(\sqrt{|g|} F^{\nu \mu} P_{\xi}\right) .
\end{aligned}
$$


As expected, locally, one can find an antisymmetric $J^{\alpha \mu}$ such that ${ }^{7}$

$$
J^{\mu}=\partial_{\alpha} J^{\alpha \mu}
$$

and, restoring the overall normalization factor of $\left(16 \pi G_{N}^{(d)}\right)^{-1}$ in the action, it is given by

$$
J^{\alpha \mu}=-4 \nabla_{\beta} P^{\alpha \mu \beta \nu} \xi_{\nu}+2 P^{\alpha \mu \beta \nu} \nabla_{\beta} \xi_{\nu}-\frac{1}{16 \pi G_{N}^{(d)}} \sqrt{|g|} F^{\alpha \mu} P_{\xi} .
$$

Now, for a Killing vector that generates an isometry which also leaves invariant the Maxwell field so we can use the momentum-map equation (1.11)

$$
\begin{aligned}
J^{\mu} & =\left(2 P^{\mu \alpha \beta \gamma} R_{\alpha \beta \gamma}^{\nu}-4 \nabla_{\alpha} \nabla_{\beta} P^{\alpha \mu \beta \nu}\right) k_{\nu}-\frac{1}{16 \pi G_{N}^{(d)}} \partial_{\nu}\left(\sqrt{|g|} F^{\nu \mu} P_{k}\right) \\
& =\mathcal{L} k^{\mu}
\end{aligned}
$$

where we have used the equations of motion and the momentum-map equation

Generically, this current does not vanish on-shell because the Lagrangian is not guaranteed to vanish on-shell. The fact that it vanishes in pure Einstein gravity is what makes it so easy to find the Komar integral in that case.

In the case of general Lovelock theories, where this problem arises even in absence of matter couplings one may try to include volume terms to the Komar surface integral, as proposed in ref. [8]. There is, however, another possibility inspired in the solution given in the same reference to the cosmological constant term: we can modify $J^{\alpha \mu}$ to absorb that term

$$
\tilde{J}^{\alpha \mu}=J^{\alpha \mu}-2 \omega^{\alpha \mu},
$$

where $\omega^{\alpha \mu}$ is a generalization of the Killing potential (density) introduced in ref. [8] defined by

$$
\partial_{\alpha} \omega^{\alpha \mu}=\frac{1}{2} \mathcal{L} k^{\mu},
$$

and whose local existence is guaranteed by the Killing equation and the symmetry condition

$$
k^{\mu} \partial_{\mu} \mathcal{L}=0
$$

which must be satisfied if the diffeomorphism generated by $k^{\mu}$ is a symmetry of the complete solution.

We arrive, then, to the generalization of the Komar integral we were looking for:

$$
\begin{aligned}
\mathcal{K}\left(\Sigma^{d-2}\right) & \equiv \frac{(-1)^{d-1}}{2} \int_{\Sigma^{d-2}} \frac{d^{d-2} \Sigma_{\alpha \mu}}{\sqrt{|g|}} \tilde{J}^{\alpha \mu} \\
& =(-1)^{d-1} \int_{\Sigma^{d-2}} \frac{d^{d-2} \Sigma_{\alpha \mu}}{\sqrt{|g|}}\left\{P^{\alpha \mu \beta \nu} \nabla_{\beta} k_{\nu}-2 \nabla_{\beta} P^{\alpha \mu \beta \nu} k_{\nu}-\frac{1}{32 \pi G_{N}^{(d)}} \sqrt{|g|} F^{\alpha \mu} P_{k}-\omega^{\alpha \mu}\right\} .
\end{aligned}
$$

Although we have only considered one matter field, it is not difficult to adapt this formula to include an uncharged scalar field with a scalar potential or a cosmological constant term, which will only contribute to the Killing potential. We are going to study a few examples in section 2 .

\footnotetext{
${ }^{7}$ The detailed calculation can be found in the appendix.
} 


\section{Examples}

\subsection{General relativity in presence of a cosmological constant}

The action of this theory is

$$
S[g]=\frac{1}{16 \pi G_{N}^{(d)}} \int d^{d} x \sqrt{|g|}\{R-(d-2) \Lambda\} .
$$

We have normalized the cosmological constant so that the equation of motion is ${ }^{8}$

$$
R_{\mu \nu}=\Lambda g_{\mu \nu}
$$

in any dimension. This means that, on-shell,

$$
\mathcal{L} / \sqrt{|g|}=2 \Lambda .
$$

We can define $\omega^{\alpha \mu}$ by the equation

$$
\nabla_{\alpha} \omega^{\alpha \mu}=\Lambda k^{\mu}
$$

to get a simpler expression.

The $P^{\alpha \mu \beta \nu}$ associated to the Einstein-Hilbert term is

$$
P^{\alpha \mu \beta \nu}=\frac{1}{16 \pi G_{N}^{(d)}} \sqrt{|g|} g^{\alpha \mu \beta \nu}, \quad \text { where } \quad g^{\alpha \mu \beta \nu} \equiv \frac{1}{2}\left(g^{\alpha \beta} g^{\mu \nu}-g^{\alpha \nu} g^{\mu \beta}\right),
$$

and taking into account all these terms we arrive to the integral

$$
\mathcal{K}\left(\Sigma^{d-2}\right)=\frac{(-1)^{d-1}}{16 \pi G_{N}^{(d)}} \int_{\Sigma^{d-2}} d^{d-2} \Sigma_{\alpha \mu}\left[\nabla^{\alpha} \xi^{\mu}-\omega^{\alpha \mu}\right],
$$

which, up to normalization, is the integral proposed in ref. [8] and which reduces to the standard Komar integral [1] in absence of cosmological constant.

We can use this Komar integral to find the Smarr formula for Schwarzschild-(a-)DS black holes following ref. [2, 4], but we will obtain it as a particular case of the Smarr formula for cosmological Reissner-Nordström-Tangherlini black holes that we will derive in section 2.3.1.

\section{$2.2 \quad$ Lovelock gravities}

Lovelock gravities are characterized by the Lagrangian densities [18]

$$
\begin{aligned}
\mathcal{L}^{k} & =\frac{\sqrt{|g|}}{16 \pi G_{N}(d)} g^{\alpha_{1} \cdots \alpha_{2 k} \beta_{1} \cdots \beta_{2 k}} R_{\alpha_{1} \alpha_{2} \beta_{1} \beta_{2}} \cdots R_{\alpha_{2 k-1} \alpha_{2 k} \beta_{2 k-1} \beta_{2 k}} \\
& =\frac{\sqrt{|g|}}{16 \pi G_{N}(d)} R_{\alpha_{1} \alpha_{2}}\left[\alpha_{1} \alpha_{2} \cdots R_{\alpha_{2 k-1} \alpha_{2 k}}{ }^{\left.\alpha_{2 k-1} \alpha_{2 k}\right]},\right.
\end{aligned}
$$

\footnotetext{
${ }^{8}$ Our cosmological constant is, therefore, related to that in ref. [9] by $\Lambda_{K R T}=\frac{(d-2)}{2} \Lambda$.
} 
where $k=0,1, \ldots$, which are non-trivial kinetic terms for the metric only for $0<2 k<d$. The case $k=0$ corresponds to the cosmological constant term of the previous example with $\Lambda=-1 /(d-2)$. It plays a non-trivial role when it is combined with other terms.

We just need to compute

$$
\begin{aligned}
P_{k}^{\mu \nu \rho \sigma} & =\frac{k \sqrt{|g|}}{16 \pi G_{N}(d)} g^{\mu \nu \alpha_{3} \cdots \alpha_{2 k} \rho \sigma \beta_{3} \cdots \beta_{2 k}} R_{\alpha_{3} \alpha_{4} \beta_{3} \beta_{4}} \cdots R_{\alpha_{2 k-1} \alpha_{2 k} \beta_{2 k-1} \beta_{2 k}} \\
& =\frac{k \sqrt{|g|}}{16 \pi G_{N}(d)} g^{\mu \nu[\rho \sigma} R_{\alpha_{3} \alpha_{4}}{ }^{\alpha_{3} \alpha_{4}} \cdots R_{\alpha_{2 k-1} \alpha_{2 k}}{ }^{\left.\alpha_{2 k-1} \alpha_{2 k}\right]}, \\
\nabla_{\mu} P^{\mu \nu \rho \sigma} & =0
\end{aligned}
$$

to get the equations of motion (up to an overall factor)

$$
k \mathcal{G}^{k \mu \nu}-\frac{1}{2} g^{\mu \nu} \mathcal{G}_{\rho}^{k \rho}=0,
$$

where

$$
\begin{aligned}
\mathcal{G}^{k \mu \nu} & \equiv g^{\mu \rho[\sigma \tau} R_{\alpha_{3} \alpha_{4}}{ }^{\alpha_{3} \alpha_{4}} \cdots R_{\alpha_{2 k-1} \alpha_{2 k}{ }^{\left.\alpha_{2 k-1} \alpha_{2 k}\right]} R_{\rho \sigma \tau}^{\nu}}, \\
\Rightarrow \quad \mathcal{G}_{\rho}^{k \rho} & =\frac{16 \pi G_{N}{ }^{(d)}}{\sqrt{|g|}} \mathcal{L}^{k} .
\end{aligned}
$$

The trace is proportional to the Lagrangian and, therefore, the equations of motion are equivalent to

$$
\mathcal{G}^{k \mu \nu}=0,
$$

which means that the Lagrangian vanishes on-shell. This leads immediately to

$$
\mathcal{K}^{k}\left(\Sigma^{d-2}\right)=\frac{(-1)^{d-1} k}{16 \pi G_{N}(d)} \int_{\Sigma^{d-2}} d^{d-2} \Sigma_{\alpha_{1} \alpha_{2}} \nabla^{\left[\alpha_{1}\right.} \xi^{\alpha_{2}} R_{\alpha_{3} \alpha_{4}}{ }^{\alpha_{3} \alpha_{4}} \cdots R_{\alpha_{2 k-1} \alpha_{2 k}}{ }^{\left.\alpha_{2 k-1} \alpha_{2 k}\right]},
$$

which is, up to normalization, the Komar integral proposed in ref. [8].

If we consider a linear combination of Lovelock terms with constant coefficients $\alpha_{k}{ }^{9}$

$$
\mathcal{L}=\sum_{k=0} \alpha_{k} \mathcal{L}^{k}
$$

the equations of motion are linear combinations of the $k$ th equations of motion

$$
\sum_{k} \alpha_{k}\left[\mathcal{G}^{k \mu \nu}-\frac{1}{2 k} g^{\mu \nu} \mathcal{G}_{\rho}^{k \rho}\right]=0
$$

and the trace, which is not proportional to the Lagrangian anymore, gives the equation

$$
\mathcal{L}^{\text {on-shell }} \frac{2}{d} \sum_{k=0} k \alpha_{k} \mathcal{L}^{k}
$$

\footnotetext{
${ }^{9}$ Now, as we said, the $k=0$ term becomes relevant and the constant $\alpha_{0}=-(d-2) \Lambda$.
} 
This relation can be used to eliminate one term from $\mathcal{L}$, typically the one of highest order, $k=m$. If $m \neq d / 2$, the result is

$$
\mathcal{L} \stackrel{\text { on-shell }}{=} \frac{2}{d} \sum_{k}^{m \neq d / 2} \frac{2(m-k)}{2 m-d} \alpha_{k} \mathcal{L}^{k}
$$

If $m=d / 2$, the highest order term is topological and we can eliminate the next one, of order $k=m-1$. The result is

$$
\mathcal{L}^{\text {on-shell }}-\sum_{k}^{d / 2} 2(d-2-2 k) \alpha_{k} \mathcal{L}^{k} .
$$

The Komar integral for these theories takes, then, the form

$$
\mathcal{K}\left(\Sigma^{d-2}\right)=\left\{\begin{array}{l}
\sum_{k}^{m \neq d / 2} \alpha_{k} \mathcal{K}^{k}\left(\Sigma^{d-2}\right)+(-1)^{d} \sum_{k}^{m \neq d / 2} \frac{2(m-k)}{2 m-d} \alpha_{k} \int_{\Sigma^{d-2}} d^{d-2} \Sigma_{\alpha \mu} \omega^{k \alpha \mu}, \\
\sum_{k}^{m=d / 2} \alpha_{k} \mathcal{K}^{k}\left(\Sigma^{d-2}\right)+(-1)^{d-1} \sum_{k}^{d / 2} 2(d-2-2 k) \alpha_{k} \int_{\Sigma^{d-2}} d^{d-2} \Sigma_{\alpha \mu} \omega^{k \alpha \mu},
\end{array}\right.
$$

where the $\omega^{k \alpha \mu}$ satisfy the equations

$$
\nabla_{\alpha} \omega^{k \alpha \mu}=\frac{16 \pi G_{N}{ }^{(d)}}{\sqrt{|g|}} \mathcal{L}^{k}
$$

where each of the Lagrangian densities $\mathcal{L}^{k}$ has to be evaluated on-shell.

Using these Komar integrals we can recover the results of refs. [2, 4] on the Smarr formula for black holes in these theories. We refer the reader to those articles for further details.

\subsection{Dilaton gravity}

The action of dilaton gravity, to which we have added a scalar potential, is given (in the Einstein frame) by

$$
S[g, A, \phi]=\frac{1}{16 \pi G_{N}^{(d)}} \int d^{d} x \sqrt{|g|}\left\{R+2(\partial \phi)^{2}-\frac{1}{4} e^{-2 a \phi} F^{2}-V(\phi)\right\},
$$

where $a$ is a constant parameter. This example includes the one considered in section 2.1 if we set to zero the scalar and Maxwell fields and replace the scalar potential by $(d-2) \Lambda$.

$P^{\mu \nu \rho \sigma}$ is, again, the one associated to the Einstein-Hilbert term eq. (2.5). The scalar field has no gauge freedom and, therefore,

$$
\mathcal{K}\left(\Sigma^{d-2}\right)=\frac{(-1)^{d-1}}{16 \pi G_{N}^{(d)}} \int_{\Sigma^{d-2}} d^{d-2} \Sigma_{\alpha \mu}\left[\nabla^{\alpha} \xi^{\mu}-\omega^{\alpha \mu}-\frac{1}{2} e^{-2 a \phi} F^{\alpha \mu} P_{k}\right],
$$

where $P_{k}$ satisfies the same momentum-map equation (1.11) but, now, $\omega^{\alpha \mu}$ satisfies the equation

$$
\nabla_{\alpha} \omega^{\alpha \mu}=\frac{8 \pi G_{N}^{(d)}}{\sqrt{|g|}} \mathcal{L} k^{\mu}
$$


where the Lagrangian

$$
\frac{8 \pi G_{N}^{(d)}}{\sqrt{|g|}} \mathcal{L}=\frac{1}{2}\left\{R+2(\partial \phi)^{2}-\frac{1}{4} e^{-2 a \phi} F^{2}-V(\phi)\right\},
$$

has to be evaluated on-shell.

The trace of the Einstein equation gives

$$
R+2(\partial \phi)^{2}=\frac{(d-4)}{4(d-2)} e^{-2 a \phi} F^{2}+\frac{d}{(d-2)} V(\phi),
$$

and the on-shell Lagrangian is given by

$$
\frac{8 \pi G_{N}^{(d)}}{\sqrt{|g|}} \mathcal{L} \stackrel{\text { on-shell }}{=}-\frac{1}{2(d-2)} e^{-2 a \phi} F^{2}+\frac{1}{(d-2)} V(\phi) .
$$

Non-trivial asymptotically-De Sitter and anti-De Sitter dilaton black holes with an ad hoc dilaton potential in 4 and higher dimensions have been constructed in refs. [19] and [20], respectively. They are quite complicated to handle and, therefore, we will just consider the $d$-dimensional cosmological Reissner-Nordström-Tangherlini black holes (more often known as $d$-dimensional Reissner-Nordström-(anti-)De Sitter black holes) [21]. In section 2.4 we will consider also Schwarzschild-(a-)DS solutions.

\subsubsection{Cosmological Reissner-Nordström-Tangherlini black holes}

These solutions take the simple form

$$
\begin{aligned}
d s^{2} & =W d t^{2}-W^{-1} d r^{2}-r^{2} d \Omega_{(d-2)}^{2} \\
W & =1-\frac{2 m}{r^{d-3}}+\left(\frac{q}{r^{d-3}}\right)^{2}-\frac{\Lambda}{(d-1)} r^{2} \\
A & =\sqrt{\frac{2(d-2)}{(d-3)}} \frac{q}{r^{d-3}} d t
\end{aligned}
$$

where the parameters $m$ and $q$, introduced to simplify the expressions, are related to the ADM mass, $M$ and to the canonically-normalized electric charge, $Q$, by

$$
m=\gamma M, \quad q=\sqrt{\frac{2(d-2)}{(d-3)}} \gamma Q, \quad \gamma \equiv \frac{8 \pi G_{N}^{(d)}}{(d-2) \omega_{(d-2)}} .
$$

We are going to focus on the aDS $(\Lambda<0)$ case for simplicity, since in that case there is only one horizon at $r=r_{h}$, which is the value of the $r$ at which $W\left(r_{h}\right)=0$.

In order to find the Smarr formula for these black holes, following ref. [2, 4], we integrate the divergence of the integrand of the Komar integral (which vanishes identically, by construction) on a hypersurface whose boundary is the disjoint union of a spatial section of the event horizon and spatial infinity. Stokes' theorem tells us that the Komar integrals over the two disjoint components of the boundary must be equal. This is the basis of the Smarr formula. Let us compute each of these integrals. 
First of all, the Komar integral can be obtained from eq. (2.21) and reads

$$
\mathcal{K}\left(\Sigma^{d-2}\right)=\frac{(-1)^{d-1}}{8 \pi G_{N}^{(d)}} \int_{\Sigma^{d-2}} d^{d-2} \Sigma_{\alpha \mu}\left[\nabla^{\alpha} \xi^{\mu}-\omega^{\alpha \mu}-\frac{1}{4} F^{\alpha \mu} P_{k}\right]
$$

where $\omega^{\alpha \mu}$ satisfies

$$
\nabla_{\alpha} \omega^{\alpha \mu}=\left[-\frac{1}{4(d-2)} F^{2}+\Lambda\right] k^{\mu}
$$

and where $k^{\mu}=\delta^{\mu}{ }_{t}$. This equation reduces to

$$
\frac{1}{r^{d-2}}\left(r^{d-2} \omega^{r t}\right)^{\prime}=\frac{(d-3) q^{2}}{r^{2(d-2)}}+\Lambda
$$

and is solved by

$$
-\omega^{t r}=-\frac{q^{2}}{r^{2(d-3)+1}}+\frac{\Lambda}{(d-1)} r+\alpha r_{h}\left(\frac{r_{h}}{r}\right)^{d-2},
$$

where the last term has been added, using the notation of ref. [8], to reflect the possibility of adding the dual of an exact $(d-2)$-form to $\omega$.

The momentum map equation (1.11) is solved by $P_{k}=A_{t}$ and

$$
-\frac{1}{2} F^{t r} P_{k}=\frac{(d-2) q^{2}}{r^{2(d-3)+1}} .
$$

Finally,

$$
\nabla^{[t} k^{r]}=\frac{1}{2} W^{\prime}=\frac{(d-3) m}{r^{d-2}}-\frac{(d-2) q^{2}}{2 r^{2(d-3)+1}}-\frac{\Lambda}{(d-1)} r,
$$

and, integrating over a sphere of constant radius $r$

$$
(-1)^{d-1} \int_{S_{r}^{d-2}} d^{d-2} \Sigma_{t r}=\omega_{(d-2)} r^{d-2},
$$

we have

$$
\begin{aligned}
\mathcal{K}\left(S_{r}^{d-2}\right) & =\frac{(-1)^{d-1}}{8 \pi G_{N}^{(d)}} \int_{S_{r}^{d-2}} d^{d-2} \Sigma_{t r}\left[\frac{(d-3) m}{r^{d-2}}+\frac{(d-4) q^{2}}{2(d-3) r^{2(d-3)+1}}+\alpha r_{h}\left(\frac{r_{h}}{r}\right)^{d-2}\right] \\
& =\left[\frac{(d-3) M}{(d-2)}+\frac{(d-4) \gamma Q^{2}}{(d-3)^{2} r^{(d-3)}}+\frac{\alpha r_{h}^{d-1}}{(d-2) \gamma}\right] \stackrel{r \rightarrow \infty}{\longrightarrow} \frac{(d-3) M}{(d-2)}+\frac{\alpha r_{h}^{d-1}}{(d-2) \gamma} .
\end{aligned}
$$

The integral on the horizon at $r=r_{h}$ is easy to compute realizing that

$$
\frac{1}{2} W^{\prime}\left(r_{h}\right)=\kappa,
$$

where $\kappa$ is the surface gravity. Then,

$$
\begin{aligned}
\mathcal{K}\left(S_{r_{h}}^{d-2}\right) & =\frac{\omega_{(d-2)} r_{h}^{d-2}}{8 \pi G_{N}^{(d)}}\left[\kappa+\frac{(d-3) q^{2}}{r_{h}^{2(d-3)+1}}+\frac{\Lambda}{(d-1)} r_{h}+\alpha r_{h}\right] \\
& =\frac{\kappa A}{8 \pi G_{N}^{(d)}}+\frac{(d-3)}{(d-2)} \Phi Q-2 \frac{\Theta \Lambda}{8 \pi G_{N}^{(d)}}+\frac{\alpha r_{h}^{d-1}}{(d-2) \gamma},
\end{aligned}
$$


where

$$
\begin{aligned}
& \Phi \equiv P_{k}\left(r_{h}\right)=\sqrt{\frac{2(d-2)}{(d-3)}} \frac{q}{r_{h}^{d-3}}, \\
& \Theta \equiv \frac{1}{2} \frac{\omega_{(d-2)} r_{h}^{d-1}}{d-1},
\end{aligned}
$$

are the electric potential of the black-hole horizon and the thermodynamic potential conjugate to the cosmological constant, respectively.

Equating $\mathcal{K}\left(S_{\infty}^{d-2}\right)$ and $\mathcal{K}\left(S_{r_{h}}^{d-2}\right)$ we arrive at the Smarr formula

$$
\frac{(d-3) M}{(d-2)}=\frac{\kappa A}{8 \pi G_{N}^{(d)}}+\frac{(d-3)}{(d-2)} \Phi Q-2 \frac{\Theta \Lambda}{8 \pi G_{N}^{(d)}} .
$$

Observe that correct factors in the $\Phi Q$ term are obtained only when the contribution from the $F^{2}$ term to $\omega^{\alpha \mu}$ and contribution from the $F^{\alpha \mu} P_{k}$ are taken into account.

\section{$2.4 f(R)$ gravity}

$f(R)$ gravities, ${ }^{10}$ although equivalent to a Brans-Dicke theory with scalar potential, can also be used to illustrate the use of the Komar integral eq. (1.28). It will also help us to make an interesting point on black-hole thermodynamics.

These theories are defined by a Lagrangian density of the form

$$
\mathcal{L}=\frac{\sqrt{|g|}}{16 \pi G_{N}^{(d)}} f(R)
$$

where $f(R)$ is a function of the Ricci scalar. We just need to compute

$$
\begin{aligned}
P^{\alpha \mu \beta \nu} & =\frac{\sqrt{|g|}}{16 \pi G_{N}^{(d)}} g^{\alpha \mu \beta \nu} f^{\prime}, \\
\nabla_{\rho} P^{\alpha \mu \beta \nu} & =\frac{\sqrt{|g|}}{16 \pi G_{N}^{(d)}} g^{\alpha \mu \beta \nu} \nabla_{\rho} f^{\prime},
\end{aligned}
$$

where $f^{\prime}$ is the derivative of the function $f$ with respect to its argument.

Then, eq. (1.5a) gives the Einstein equations

$$
\frac{16 \pi G_{N}^{(d)}}{\sqrt{|g|}} \frac{\delta S}{\delta g_{\mu \nu}}=\frac{1}{2} g^{\mu \nu} f-f^{\prime} R^{\mu \nu}+\left(g^{\mu \nu} \nabla^{2}-\nabla^{\mu} \nabla^{\nu}\right) f^{\prime},
$$

and eq. (1.28) gives the Komar integral

$$
\mathcal{K}\left(\Sigma^{d-2}\right)=\frac{(-1)^{d-1}}{16 \pi G_{N}^{(d)}} \int_{\Sigma^{d-2}} d^{d-2} \Sigma_{\alpha \mu}\left\{f^{\prime} \nabla^{\alpha} k^{\mu}-2 \nabla^{\alpha} f^{\prime} k^{\mu}-\omega^{\alpha \mu}\right\}
$$

where

$$
\nabla_{\alpha} \omega^{k \alpha \mu}=\frac{16 \pi G_{N}(d)}{\sqrt{|g|}} \mathcal{L}
$$

\footnotetext{
${ }^{10}$ For a review with many references, see ref. [22].
} 
where the Lagrangian density has to be evaluated on-shell. The trace of the equation of motion (2.42) gives the relation

$$
\frac{d}{2} f-f^{\prime} R+(d-2) \nabla^{2} f^{\prime}=0
$$

which, as in the Lovelock case, can be used to eliminate terms in $\mathcal{L}$.

Let us consider a simple model, described by a second degree polynomial:

$$
f(R)=-(d-2) \Lambda+R+\alpha_{2} R^{2}
$$

where the zeroth and linear (Einstein-Hilbert) terms have the standard normalization.

The cosmological Schwarzschild-Tangherlini metrics [21] given in eqs. (2.26) setting $q=0$, are known to be solutions of these theories. The constants $m$ and $\lambda$ are, essentially, the mass and the cosmological constant in absence of the $R^{2}$ term, according to eqs. (2.27). In this theory, though, there is a more complicated relation between $m$ and $\lambda$ and $M$ and $\Lambda$.

$$
R_{\mu \nu}=\lambda g_{\mu \nu}
$$

where $\lambda$ is the effective cosmological constant, to be distinguished from the cosmological constant $\Lambda$ in the action. Substituting this condition in eq. (2.45) we find (only for $d \neq 4$ and $\alpha_{2} \neq 0$ )

$$
\lambda=-\frac{1}{\beta}\{1 \pm \sqrt{1+2 \Lambda \beta}\}, \quad \text { where } \quad \beta \equiv 2 \alpha_{2} d(d-4) /(d-2) .
$$

For this theory and these solutions, the Komar integral eq. (2.43) over a $S^{d-2}$ of radius $r$

$$
\mathcal{K}\left(S_{r}^{d-2}\right)=\frac{\omega_{(d-2)}}{8 \pi G_{N}^{(d)}}\left(1+2 \alpha_{2} d \lambda\right)\left\{\frac{r^{d-2}}{2} W^{\prime}+\frac{\lambda r^{d-1}}{(d-1)}+\alpha r_{h}^{d-1}\right\} .
$$

The terms that diverge at infinity cancel identically and we get

$$
\mathcal{K}\left(S_{\infty}^{d-2}\right)=\frac{(d-3)}{(d-2)} M+\frac{\alpha r_{h}^{d-1}}{(d-2) \gamma}\left(1+2 \alpha_{2} d \lambda\right)
$$

where, now

$$
m=\gamma M /\left(1+2 \alpha_{2} d \lambda\right) .
$$

Over the horizon

$$
\mathcal{K}\left(S_{r_{h}}^{d-2}\right)=S T+\frac{\omega_{(d-2)} r_{h}^{d-1}}{8 \pi G_{N}^{(d)}(d-1)}\left(1+2 \alpha_{2} d \lambda\right) \lambda+\frac{\alpha r_{h}^{d-1}}{(d-2) \gamma}\left(1+2 \alpha_{2} d \lambda\right),
$$

where $T=\frac{\kappa}{2 \pi}$ and $S$, Wald's entropy, is given by

$$
S=\left(1+2 \alpha_{2} d \lambda\right) \frac{\omega_{(d-2) r_{h}^{d-2}}}{4 G_{N}^{(d)}}
$$


and we finally arrive at

$$
\frac{(d-3)}{(d-2)} M=S T+\frac{\omega_{(d-2)} r_{h}^{d-1}}{8 \pi G_{N}^{(d)}(d-1)}\left(1+2 \alpha_{2} d \lambda\right) \lambda .
$$

The second term in the right-hand side can be interpreted in the same spirit as in the Lovelock case, in terms of two contributions associated to the dimensionful parameters that appear in the action, $\Lambda$ and $\alpha_{2}$. These will be identified with thermodynamical variables ("pressures") and their conjugate potentials ("volumes") $\Theta_{\Lambda}$ and $\Theta_{\alpha_{2}}$

$$
\frac{(d-3) M}{(d-2)}=S T-2 \frac{\Theta_{\Lambda} \Lambda\left(1+2 \alpha_{2} d \lambda\right)}{8 \pi G_{N}^{(d)}}+2 \frac{\Theta_{\alpha_{2}} \alpha_{2}\left(1+2 \alpha_{2} d \lambda\right)}{8 \pi G_{N}^{(d)}},
$$

with $\Theta_{\Lambda}$ and $\Theta_{\alpha_{2}}$ given by

$$
\begin{aligned}
\Theta_{\Lambda} & \equiv \frac{\Theta}{1+2 \alpha_{2} d \lambda}, \\
\Theta_{\alpha_{2}} & \equiv-\frac{d^{2} \lambda^{2} \Theta}{(d-2)\left(1+2 \alpha_{2} d \lambda\right)} .
\end{aligned}
$$

These "volumes" are the contributions of the cosmological and $R^{2}$ terms to the Komar integral on the horizon.

It is interesting to recover the same results in the equivalent Brans-Dicke theory, whose Lagrangian density has the form

$$
\mathcal{L}=\frac{\sqrt{|g|}}{16 \pi G_{N}^{(d)}}[\phi R-V(\phi)]
$$

with

$$
\begin{aligned}
\phi & =f^{\prime}(R)=1+2 \alpha_{2} R, \\
V(\phi) & =R f^{\prime}(R)-f(R)=(d-2) \Lambda+\frac{1}{4 \alpha_{2}}(\phi-1)^{2} .
\end{aligned}
$$

It is convenient to rescale the metric to the Einstein frame. If we want the rescaling to relate asymptotically-flat or asymptotically-aDS metrics with the same normalization, the rescaling has to be performed with $\phi / \phi_{\infty}$, where $\phi_{\infty}$ is the asymptotic value of the scalar $\left(1+2 \alpha_{2} d \lambda\right.$ in our case). First, we rewrite the Lagrangian as

$$
\mathcal{L}=\frac{\sqrt{|g|} \phi_{\infty}}{16 \pi G_{N}^{(d)}}\left[\left(\phi / \phi_{\infty}\right) R-V(\phi) / \phi_{\infty}\right],
$$

and replace the metric $g_{\mu \nu}$ by $\left(\phi / \phi_{\infty}\right)^{-2 /(d-2)} g_{\mu \nu}$, which leads to

$$
\mathcal{L}=\frac{\sqrt{|g|} \phi_{\infty}}{16 \pi G_{N}^{(d)}}\left[R+\frac{(d-2)}{(d-2)}\left(\partial \ln \left(\phi / \phi_{\infty}\right)\right)^{2}-2 \frac{(d-2)}{(d-2)} \nabla^{2} \ln \left(\phi / \phi_{\infty}\right)-\mathcal{V}(\phi)\right]
$$

where

$$
\mathcal{V}(\phi) \equiv \phi_{\infty}^{2 /(d-2)}\left[(d-2) \Lambda \phi^{-d /(d-2)}+\frac{1}{4 \alpha_{2}} \phi^{-d /(d-2)}(\phi-1)^{2}\right]
$$


is the new scalar potential which, not surprisingly, is extremized by $\phi=1+2 \alpha_{2} d \lambda$. This is the value of $\phi_{\infty}$ and we see that the effective cosmological constant is $\lambda,{ }^{11}$ and that the effective Newton constant in this theory is just $G_{N}^{(d)} / \phi_{\infty}$. The mass and the entropy, measured in this theory, take, then, the same value as the mass and entropy measured in the higher-order $f(R)$ theory, eqs. (2.51) and (2.53).

We can use our previous results to find the Smarr formula through the Komar integral for this theory. The on-shell Lagrangian is proportional to the scalar potential and we could express it as in eq. (2.39) with $\Lambda$ replaced by the effective cosmological constant $\lambda$. However, it is more natural to express it in terms of the original dimensionful constants that define the theory, namely $\Lambda$ and $\alpha_{2}$, as in eq. (2.55).

In the Brans-Dicke form of the theory, the "volumes" can be computed as surface or volume integrals associated to the two terms of the potential:

$$
\begin{aligned}
\Theta_{\Lambda} & =\frac{1}{(d-2)} \int_{\mathcal{B}_{r_{h}}^{d-2}} d^{d-1} \Sigma_{\mu} k^{\mu} \frac{\partial \mathcal{V}}{\partial \Lambda}, \\
\Theta_{\alpha_{2}} & =\frac{1}{(d-2)} \int_{\mathcal{B}_{r_{h}}^{d-2}} d^{d-1} \Sigma_{\mu} k^{\mu} \frac{\partial \mathcal{V}}{\partial \alpha_{2}},
\end{aligned}
$$

where $\mathcal{B}_{r_{h}}^{d-2}$ is the ball of radius $r_{h}$ whose boundary is the spatial section of the horizon we have considered.

This suggests that for potentials depending on parameters $\alpha_{k}$ of dimensions $\left[L^{k}\right]$ the Smarr formula should take the general form

$$
\frac{(d-3) M}{(d-2)}=S T+\sum_{k} k \frac{\Theta_{\alpha_{k}} \alpha_{k} \phi_{\infty}}{8 \pi G_{N}^{(d)}},
$$

with

$$
\Theta_{\alpha_{k}}=\frac{1}{(d-2)} \int_{\mathcal{B}_{r_{h}}^{d-2}} d^{d-1} \Sigma_{\mu} k^{\mu} \frac{\partial \mathcal{V}}{\partial \alpha_{k}} .
$$

This point of view should be contrasted with ethe one in ref. [23] in which all these terms are, effectively, combined in one.

\section{Discussion}

In this paper we have argued that the dimensionful constants that define scalar potentials should be treated as thermodynamical variables, by analogy with the treatment of the dimensional parameters of Lovelock theories in ref. [2] or of the parameter of the BornInfeld theory in ref. [24]. Although we have not studied directly the first law of black-hole mechanics, it is clear that one could proceed, for instance, as in ref. [25], including variations of those parameters, to derive a first law that includes them as thermodynamical parameters using Wald's formalism. A less ad hoc procedure is, nevertheless, quite desirable and work in this direction is well under way [16].

\footnotetext{
${ }^{11} \mathcal{V}\left(\phi_{\infty}\right)=(d-2) \lambda$ in our conventions.
} 
The most interesting example of theories with scalar fields and scalar potentials is provided by gauged supergravities. ${ }^{12}$ Their potentials depend on coupling constants, such as gauge coupling constants and Stückelberg mass parameters, that "deform" the original theory. The implication is that those parameters, many of them codified in the so-called embedding tensor, should be considered thermodynamical variables. These parameters can, in their turn, be related to potentials that couple to branes [28, 29] which raises the possibility of intriguing connections between those branes and the thermodynamical variables which deserve to be studied [16].

In section 2.3.1 we have considered charged black holes in general $d$ dimensions and we have not considered the magnetically charged ones that can exist in $d=4$. Those solutions can easily be obtained using electric-magnetic duality. In particular we should replace $q^{2}$ by $q^{2}+p^{2}$, where $p$ is (up to constants) the magnetic charge in the metric and, correspondingly, $\Phi Q$ by $\Phi Q+\Xi P$, where $\Xi$ is the magnetic potential on the horizon and $P$ the magnetic charge, in the Smarr formula. However, had we considered those solutions, and had we used the Komar formula eq. (1.28) we would have arrived to a different (wrong) Smarr formula. This is due to the fact that Wald's formalism can only account for variations of electric charges ${ }^{13}$ in the first law, a problem we have pointed out in ref. [13] and which shows in the expressions for the first law obtained in refs. [12-14].

Although they do not contribute to the Smarr fomulae, ${ }^{14}$ scalars do contribute to the first law [30, 31] and Wald's formalism cannot account for their contributions, either. A common solution to the two problems that we have mentioned (and, perhaps, to properly include the variations of the dimensionful constants) would be to use "democratic formulations" of the theories under consideration, which include all the original ("fundamental" or "electric") fields of the theory (including deformation constants) together with their ("magnetic") duals, as in ref. [28, 32]. Work in this direction is also in progress [33].

\section{Acknowledgments}

The author would like to thank R.B. mann for the course on black-hole chemistry given at the IFT and P. Bueno, P. Cano, P. Meessen and D. Pereñíguez for useful conversations. This work has been supported in part by the MCIU, AEI, FEDER (UE) grant PGC2018095205-B-I00 and by the Spanish Research Agency (Agencia Estatal de Investigación) through the grant IFT Centro de Excelencia Severo Ochoa SEV-2016-0597. TO wishes to thank M.M. Fernández for her permanent support.

\footnotetext{
${ }^{12}$ See ref. [26] for a quite complete review and ref. [27] for a more pedagogical introduction.

${ }^{13}$ Those which would be naturally carried by objects coupling to the potentials appearing in the action. The Maxwell field, for instance, couples to electrically-charged point particles only. Magentic monopoles couple to the dual of the Maxwell field.

${ }^{14}$ This can be seen using the scaling argument reviewed, for instance, in ref. [2].
} 


\section{A Determination of $\boldsymbol{J}^{\alpha \mu}$}

Let us consider the first term in eq. (1.21). Expanding the symmetrizer and using the Ricci identity

$$
\left[\nabla_{\mu}, \nabla_{\nu}\right] \xi_{\rho}=-R_{\mu \nu \rho \sigma} \xi^{\sigma}
$$

we get

$$
\begin{aligned}
P^{\mu \alpha \beta \gamma} \nabla_{\beta} \nabla_{(\alpha} \xi_{\gamma)} & =\frac{1}{2} P^{\mu \alpha \beta \gamma} \nabla_{\beta} \nabla_{\alpha} \xi_{\gamma}+\frac{1}{2} P^{\mu \alpha \beta \gamma} \nabla_{\beta} \nabla_{\gamma} \xi_{\alpha} \\
& =\frac{1}{2} P^{\mu \alpha \beta \gamma}\left(\nabla_{\alpha} \nabla_{\beta} \xi_{\gamma}-R_{\beta \alpha \gamma \nu} \xi^{\nu}\right)+\frac{1}{2} P^{\mu \alpha \beta \gamma}\left(-\frac{1}{2} R_{\beta \gamma \alpha \nu} \xi^{\nu}\right) \\
& =\frac{1}{2} P^{\mu \alpha \beta \gamma} \nabla_{\alpha} \nabla_{\beta} \xi_{\gamma}+\frac{1}{2} P^{\mu \alpha \beta \sigma} R_{\nu \alpha \beta \sigma},
\end{aligned}
$$

where we have used the Bianchi identity $R_{[\mu \nu \rho] \sigma}=0$ in the last step. Integrating by parts the first term twice, we get

$$
\begin{aligned}
P^{\mu \alpha \beta \gamma} \nabla_{\beta} \nabla_{(\alpha} \xi_{\gamma)}= & \nabla_{\alpha}\left(\frac{1}{2} P^{\mu \alpha \beta \gamma} \nabla_{\beta} \xi_{\gamma}\right)-\frac{1}{2} \nabla_{\alpha} P^{\mu \alpha \beta \gamma} \nabla_{\beta} \xi_{\gamma}+\frac{1}{2} P^{\mu \alpha \beta \sigma} R_{\nu \alpha \beta \sigma} \\
= & \nabla_{\alpha}\left(\frac{1}{2} P^{\mu \alpha \beta \gamma} \nabla_{\beta} \xi_{\gamma}\right)+\nabla_{\beta}\left(-\frac{1}{2} \nabla_{\alpha} P^{\mu \alpha \beta \gamma} \xi_{\gamma}\right) \\
& +\frac{1}{2} \nabla_{\beta} \nabla_{\alpha} P^{\mu \alpha \beta \gamma} \xi_{\gamma}+\frac{1}{2} P^{\mu \alpha \beta \sigma} R_{\nu \alpha \beta \sigma} \\
= & \nabla_{\alpha}\left(\frac{1}{2} P^{\mu \alpha \beta \gamma} \nabla_{\beta} \xi_{\gamma}\right)+\nabla_{\beta}\left(-\frac{1}{2} \nabla_{\alpha} P^{\mu \alpha \beta \gamma} \xi_{\gamma}\right) \\
& +\nabla_{(\beta} \nabla_{\alpha)} P^{\mu \alpha \beta \gamma} \xi_{\gamma}-\frac{1}{2} \nabla_{\alpha} \nabla_{\beta} P^{\mu \alpha \beta \gamma} \xi_{\gamma}+\frac{1}{2} P^{\mu \alpha \beta \sigma} R_{\nu \alpha \beta \sigma} \\
= & \nabla_{\alpha}\left(\frac{1}{2} P^{\mu \alpha \beta \gamma} \nabla_{\beta} \xi_{\gamma}-\frac{1}{2} \nabla_{\beta} P^{\mu \alpha \beta \gamma} \xi_{\gamma}\right)+\nabla_{\beta}\left(-\frac{1}{2} \nabla_{\alpha} P^{\mu \alpha \beta \gamma} \xi_{\gamma}\right) \\
& +\nabla_{(\beta} \nabla_{\alpha)} P^{\mu \alpha \beta \gamma} \xi_{\gamma}+\frac{1}{2} \nabla_{\beta} P^{\mu \alpha \beta \gamma} \nabla_{\alpha} \xi_{\gamma}+\frac{1}{2} P^{\mu \alpha \beta \sigma} R_{\nu \alpha \beta \sigma},
\end{aligned}
$$

where we have performed yet another integration by parts in the last step.

Now we have to use the identity eq. (1.7) in the second term of the first line

$$
\begin{aligned}
P^{\mu \alpha \beta \gamma} \nabla_{\beta} \nabla_{(\alpha} \xi_{\gamma)}= & \nabla_{\alpha}\left(\frac{1}{2} P^{\mu \alpha \beta \gamma} \nabla_{\beta} \xi_{\gamma}-\frac{1}{2} \nabla_{\beta} P^{\mu \alpha \beta \gamma} \xi_{\gamma}\right) \\
& +\nabla_{\beta}\left[\frac{1}{2} \nabla_{\alpha}\left(P^{\alpha \beta \mu \gamma}+P^{\beta \mu \alpha \gamma}\right) \xi_{\gamma}\right] \\
& -\nabla_{(\alpha} \nabla_{\beta)} P^{\mu \alpha \beta \gamma} \xi_{\gamma}+\frac{1}{2} \nabla_{\beta} P^{\mu \alpha \beta \gamma} \nabla_{\alpha} \xi_{\gamma}+\frac{1}{2} P^{\mu \alpha \beta \sigma} R_{\nu \alpha \beta \sigma} \\
= & \nabla_{\alpha}\left(\frac{1}{2} P^{\mu \alpha \beta \gamma} \nabla_{\beta} \xi_{\gamma}-\frac{1}{2} \nabla_{\beta} P^{\mu \alpha \beta \gamma} \xi_{\gamma}\right) \\
& -\nabla_{\beta}\left(\frac{1}{2} \nabla_{\alpha} P^{\beta \alpha \mu \gamma} \xi_{\gamma}\right)-\nabla_{\alpha}\left(\frac{1}{2} \nabla_{\beta} P^{\mu \alpha \beta \gamma} \xi_{\gamma}\right) \\
& +\frac{1}{2} \nabla_{\beta} P^{\mu \alpha \beta \gamma} \nabla_{\alpha} \xi_{\gamma}-\nabla_{(\alpha} \nabla_{\beta)} P^{\mu \alpha \beta \gamma} \xi_{\gamma}+\frac{1}{2} P^{\mu \alpha \beta \sigma} R_{\nu \alpha \beta \sigma}
\end{aligned}
$$




$$
\begin{aligned}
= & \nabla_{\alpha}\left(\frac{1}{2} P^{\mu \alpha \beta \gamma} \nabla_{\beta} \xi_{\gamma}-\nabla_{\beta} P^{\mu \alpha \beta \gamma} \xi_{\gamma}\right) \\
& -\frac{1}{2} \nabla_{\beta} \nabla_{\alpha} P^{\beta \alpha \mu \gamma} \xi_{\gamma} \\
& +\nabla_{\beta} P^{\beta \alpha \mu \gamma} \nabla_{(\alpha} \xi_{\gamma)}-\nabla_{(\alpha} \nabla_{\beta)} P^{\mu \alpha \beta \gamma} \xi_{\gamma}+\frac{1}{2} P^{\mu \alpha \beta \sigma} R_{\nu \alpha \beta \sigma} .
\end{aligned}
$$

Now, because of the antisymmetry of $P^{\mu \nu \rho \sigma}$ in the first two indices, we can use the Ricci identity to show that the term in the second line vanishes identically:

$$
\begin{aligned}
\nabla_{\alpha} \nabla_{\beta} P^{\alpha \beta \mu \gamma} & =\frac{1}{2}\left\{R_{\alpha \beta \delta}{ }^{\alpha} P^{\delta \beta \mu \gamma}+R_{\alpha \beta \delta^{\beta}} P^{\alpha \delta \mu \gamma}+R_{\alpha \beta \delta^{\mu}} P^{\alpha \beta \delta \gamma}+R_{\alpha \beta \delta^{\gamma}} P^{\alpha \beta \mu \delta}\right\} \\
& =\frac{1}{2}\left\{-R_{\beta \delta} P^{\delta \beta \mu \gamma}+R_{\alpha \delta} P^{\alpha \delta \mu \gamma}+R_{\alpha \beta \delta}{ }^{\mu} P^{\alpha \beta \delta \gamma}+R_{\alpha \beta \delta}{ }^{\gamma} P^{\alpha \beta \mu \delta}\right\} \\
& =R_{\alpha \beta \delta}{ }^{(\mu \mid} P^{\alpha \beta \delta \mid \gamma)} \\
& =0
\end{aligned}
$$

where we have used eq. (1.8) in the last step.

Flipping the indices $\alpha \mu$, we arrive to the identity

$$
\begin{aligned}
\nabla_{\alpha}\left(\nabla_{\beta} P^{\alpha \mu \beta \gamma} \xi_{\gamma}-\frac{1}{2} P^{\alpha \mu \beta \gamma} \nabla_{\beta} \xi_{\gamma}\right)= & P^{\mu \alpha \beta \gamma} \nabla_{\beta} \nabla_{(\alpha} \xi_{\gamma)}-\nabla_{\beta} P^{\beta \alpha \mu \gamma} \nabla_{(\alpha} \xi_{\gamma)} \\
& -\frac{1}{2} P^{\mu \alpha \beta \sigma} R_{\nu \alpha \beta \sigma}+\nabla_{(\alpha} \nabla_{\beta)} P^{\mu \alpha \beta \gamma} \xi_{\gamma},
\end{aligned}
$$

from which eq. (1.23) easily follows.

Open Access. This article is distributed under the terms of the Creative Commons Attribution License (CC-BY 4.0), which permits any use, distribution and reproduction in any medium, provided the original author(s) and source are credited.

\section{References}

[1] A. Komar, Covariant conservation laws in general relativity, Phys. Rev. 113 (1959) 934 [INSPIRE].

[2] D. Kastor, S. Ray and J. Traschen, Smarr formula and an extended first law for Lovelock gravity, Class. Quant. Grav. 27 (2010) 235014 [arXiv: 1005.5053] [INSPIRE].

[3] L. Smarr, Mass formula for Kerr black holes, Phys. Rev. Lett. 30 (1973) 71 [Erratum ibid. 30 (1973) 521] [INSPIRE].

[4] S. Liberati and C. Pacilio, Smarr formula for Lovelock black holes: a Lagrangian approach, Phys. Rev. D 93 (2016) 084044 [arXiv:1511.05446] [INSPIRE].

[5] J. Lee and R.M. Wald, Local symmetries and constraints, J. Math. Phys. 31 (1990) 725 [INSPIRE].

[6] R.M. Wald, Black hole entropy is the Noether charge, Phys. Rev. D 48 (1993) R3427 [gr-qc/9307038] [INSPIRE].

[7] V. Iyer and R.M. Wald, Some properties of Noether charge and a proposal for dynamical black hole entropy, Phys. Rev. D 50 (1994) 846 [gr-qc/9403028] [InSPIRE]. 
[8] D. Kastor, Komar integrals in higher (and lower) derivative gravity, Class. Quant. Grav. 25 (2008) 175007 [arXiv:0804.1832] [INSPIRE].

[9] D. Kastor, S. Ray and J. Traschen, Enthalpy and the mechanics of AdS black holes, Class. Quant. Grav. 26 (2009) 195011 [arXiv:0904.2765] [INSPIRE].

[10] R.B. Mann, Black holes: thermodynamics, information, and firewalls, Springer, Cham, Switzerland (2015) [INSPIRE].

[11] D. Kubiznak, R.B. Mann and M. Teo, Black hole chemistry: thermodynamics with Lambda, Class. Quant. Grav. 34 (2017) 063001 [arXiv:1608.06147] [INSPIRE].

[12] Z. Elgood, P. Meessen and T. Ortín, The first law of black hole mechanics in the Einstein-Maxwell theory revisited, JHEP 09 (2020) 026 [arXiv:2006. 02792] [INSPIRE].

[13] Z. Elgood, D. Mitsios, T. Ortín and D. Pereñíguez, The first law of heterotic stringy black hole mechanics at zeroth order in $\alpha^{\prime}$, JHEP 07 (2021) 007 [arXiv:2012.13323] [INSPIRE].

[14] Z. Elgood, T. Ortín and D. Pereñíguez, The first law and Wald entropy formula of heterotic stringy black holes at first order in $\alpha^{\prime}$, JHEP 05 (2021) 110 [arXiv:2012.14892] [INSPIRE].

[15] P. Bueno, P.A. Cano, V.S. Min and M.R. Visser, Aspects of general higher-order gravities, Phys. Rev. D 95 (2017) 044010 [arXiv:1610.08519] [InSPIRE].

[16] Z. Elgood, P. Meessen, D. Mitsios, T. Ortín and D. Pereñíguez, in preparation.

[17] T. Padmanabhan, Some aspects of field equations in generalised theories of gravity, Phys. Rev. D 84 (2011) 124041 [arXiv:1109.3846] [INSPIRE].

[18] D. Lovelock, The Einstein tensor and its generalizations, J. Math. Phys. 12 (1971) 498 [INSPIRE].

[19] C.J. Gao and S.N. Zhang, Dilaton black holes in de Sitter or anti-de Sitter universe, Phys. Rev. D 70 (2004) 124019 [hep-th/0411104] [INSPIRE].

[20] C.J. Gao and S.N. Zhang, Higher dimensional dilaton black holes with cosmological constant, Phys. Lett. B 605 (2005) 185 [hep-th/0411105] [INSPIRE].

[21] F.R. Tangherlini, Schwarzschild field in $n$ dimensions and the dimensionality of space problem, Nuovo Cim. 27 (1963) 636 [INSPIRE].

[22] T.P. Sotiriou and V. Faraoni, $f(R)$ theories of gravity, Rev. Mod. Phys. 82 (2010) 451 [arXiv:0805.1726] [INSPIRE].

[23] D. Kastor, S. Ray and J. Traschen, Black hole enthalpy and scalar fields, Class. Quant. Grav. 36 (2019) 024002 [arXiv: 1807.09801] [INSPIRE].

[24] S. Gunasekaran, R.B. Mann and D. Kubiznak, Extended phase space thermodynamics for charged and rotating black holes and Born-Infeld vacuum polarization, JHEP 11 (2012) 110 [arXiv: 1208.6251] [INSPIRE].

[25] M. Urano, A. Tomimatsu and H. Saida, Mechanical first law of black hole spacetimes with cosmological constant and its application to Schwarzschild-de Sitter spacetime, Class. Quant. Grav. 26 (2009) 105010 [arXiv: 0903.4230] [INSPIRE].

[26] M. Trigiante, Gauged supergravities, Phys. Rept. 680 (2017) 1 [arXiv:1609.09745] [INSPIRE].

[27] T. Ortín, Gravity and strings, $2^{\text {nd }}$ edition, Cambridge University Press, Cambridge, U.K. (2015). 
[28] E.A. Bergshoeff, J. Hartong, O. Hohm, M. Huebscher and T. Ortín, Gauge theories, duality relations and the tensor hierarchy, JHEP 04 (2009) 123 [arXiv:0901.2054] [INSPIRE].

[29] J. Hartong and T. Ortín, Tensor hierarchies of 5- and 6-dimensional field theories, JHEP 09 (2009) 039 [arXiv:0906.4043] [INSPIRE].

[30] G.W. Gibbons, R. Kallosh and B. Kol, Moduli, scalar charges, and the first law of black hole thermodynamics, Phys. Rev. Lett. 77 (1996) 4992 [hep-th/9607108] [INSPIRE].

[31] D. Astefanesei, R. Ballesteros, D. Choque and R. Rojas, Scalar charges and the first law of black hole thermodynamics, Phys. Lett. B 782 (2018) 47 [arXiv: 1803.11317] [INSPIRE].

[32] E. Bergshoeff, R. Kallosh, T. Ortín, D. Roest and A. Van Proeyen, New formulations of $D=10$ supersymmetry and D8-O8 domain walls, Class. Quant. Grav. 18 (2001) 3359 [hep-th/0103233] [INSPIRE].

[33] Z. Elgood, P. Meessen, D. Mitsios and T. Ortín, in preparation. 